\title{
$100 \%$ peer review in radiation oncology: is it feasible?
}

\author{
E. Martin-Garcia ${ }^{1,3}$ (D) F. Celada-Álvarez ${ }^{1} \cdot$ M. J. Pérez-Calatayud ${ }^{1} \cdot$ M. Rodriguez-Pla ${ }^{1}$ - O. Prato-Carreño ${ }^{1}$. \\ D. Farga-Albiol ${ }^{1}$ - O. Pons-Llanas ${ }^{1}$ - S. Roldán-Ortega ${ }^{1}$ - E. Collado-Ballesteros ${ }^{1}$ - F. J. Martinez-Arcelus ${ }^{1}$. \\ Y. Bernisz-Diaz ${ }^{1}$. V. A. Macias ${ }^{1}$. J. Chimeno ${ }^{2}$. J. Gimeno-Olmos ${ }^{2}$ - F. Lliso ${ }^{2}$ - V. Carmona ${ }^{2}$. J. C. Ruiz ${ }^{2}$. \\ J. Pérez-Calatayud ${ }^{2} \cdot$ A. Tormo-Micó ${ }^{1} \cdot$ A. J. Conde-Moreno' ${ }^{1}$
}

Received: 16 December 2019 / Accepted: 12 May 2020 / Published online: 15 June 2020

(c) Federación de Sociedades Españolas de Oncología (FESEO) 2020

\begin{abstract}
Purpose Peer review has been proposed as a strategy to ensure patient safety and plan quality in radiation oncology. Despite its potential benefits, barriers commonly exist to its optimal implementation in daily clinical routine. Our purpose is to analyze peer-review process at our institution.

Methods and materials Based on our group peer-review process, we quantified the rate of plan changes, time and resources needed for this process. Prospectively, data on cases presented at our institutional peer-review conference attended by physicians, resident physicians and physicists were collected. Items such as time to present per case, type of patient (adult or pediatric), treatment intent, dose, aimed technique, disease location and receipt of previous radiation were gathered. Cases were then analyzed to determine the rate of major change, minor change and plan rejection after presentation as well as the median time per session.

Results Over a period of 4 weeks, 148 cases were reviewed. Median of attendants was six physicians, three in-trainingphysicians and one physicist. Median time per session was 38 (4-72) minutes. 59.5\% of cases presented in 1-4 min, 32.4\% in $5-9 \mathrm{~min}$ and $8.1 \%$ in $\geq 10 \mathrm{~min}$. $79.1 \%$ of cases were accepted without changes, $11.5 \%$ with minor changes, $6 \%$ with major changes and 3.4\% were rejected with indication of new presentation. Most frequent reason of change was contouring corrections $(53.8 \%)$ followed by dose or fractionation $(26.9 \%)$.

Conclusion Everyday group consensus peer review is an efficient manner to recollect clinical and technical data of cases presented to ensure quality radiation care before initiation of treatment as well as ensuring department quality in a feedback team environment. This model is feasible within the normal operation of every radiation oncology Department.
\end{abstract}

Keywords Peer review · Quality assurance $\cdot$ Radiation oncology $\cdot$ Patient safety $\cdot$ Treatment planning $\cdot$ Treatment quality

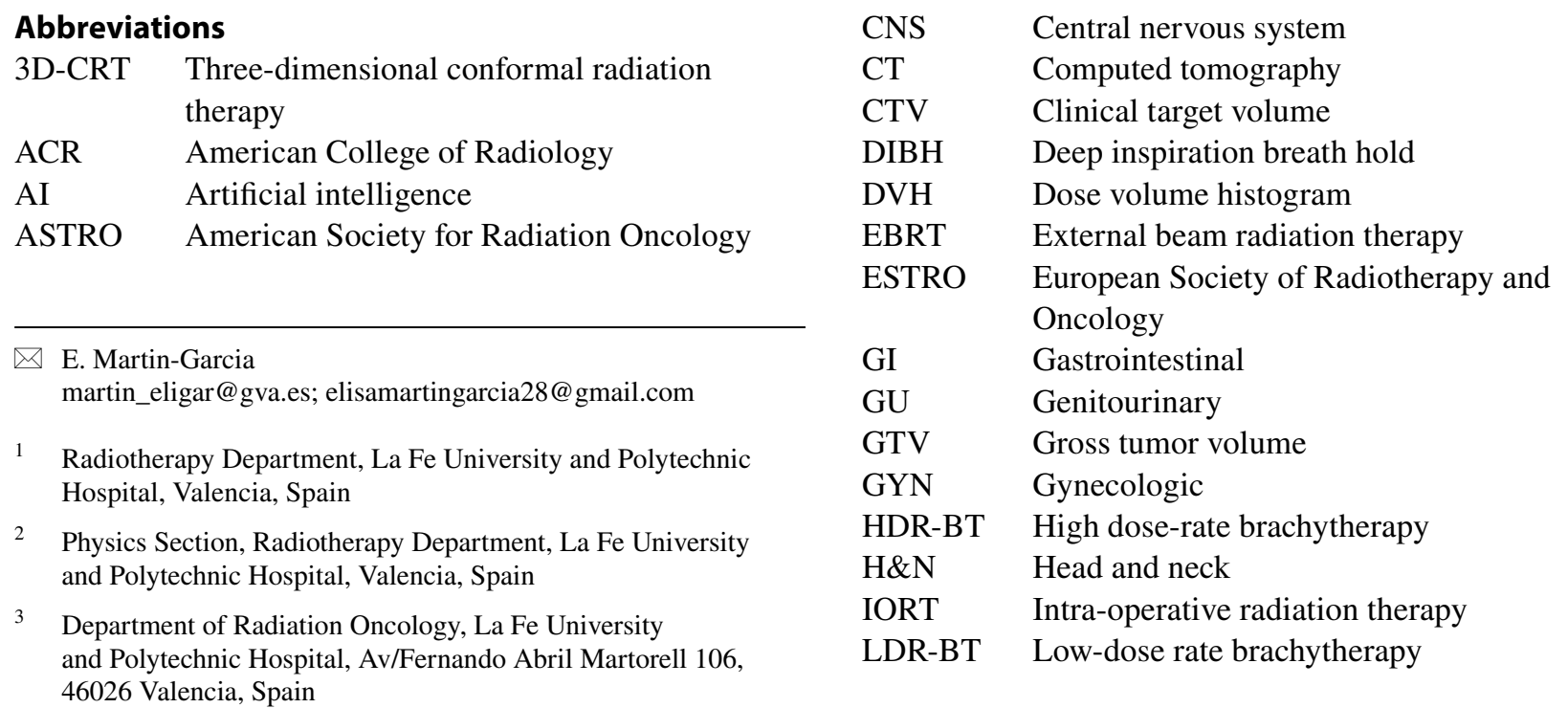




$\begin{array}{ll}\text { MRI } & \text { Magnetic resonance imaging } \\ \text { OAR } & \text { Organ at risk } \\ \text { PET/CT } & \text { Positron-emission tomography } \\ \text { PTV } & \text { Planning target volume } \\ \text { QA } & \text { Quality assurance } \\ \text { RANZCR } & \text { Royal Australian and New Zealand College } \\ & \text { Radiologists } \\ \text { SBRT } & \text { Stereotactic body radiation therapy } \\ \text { SEOR } & \text { Spanish Society of Radiation Oncology } \\ \text { SR } & \text { Stereotactic radiosurgery } \\ \text { TBI } & \text { Total body irradiation } \\ \text { TV } & \text { Target volumes } \\ \text { VMAT } & \text { Volumetric modulated arc therapy }\end{array}$

\section{Introduction}

Assuring quality of care is a safety challenge within Radiation Oncology related to the evolving growth of technology and the multistep planning process in this field.

Individual decision making among radiation oncology physicians and the complexity of radiation treatment plans can also contribute to potential errors in this process. Target volumes and normal tissue delineations represent an important source of those errors as well as treatment prescription (total dose and dose fractionation), especially for hypofractionated schemes and SBRT plans.

As such, an adequate peer-review process involving plan evaluation in a feedback environment from a multidisciplinary team is an effective strategy for assuring plan quality and patient safety recommended by professional organizations such as ASTRO, ACR and RANZCR [1-3].

Several reports have proven the benefit of peer-review process into facilitating treatment plans but also have enumerated a list of barriers to its effective implementation and efficiency such as scheduling, workload, time dedicated to peer review, equipment, repetition, the attendance by key personnel and distraction from clinic time with patients [1, 4-6]. In addition, there is still little guidance and limited published research about the frequency, mechanics or metrics of peer review in radiation oncology from professional organizations $[1,2]$ so nowadays, each radiation oncology department should identify and mitigate those barriers to an effective application of peer review to its individual process and workflow.

Currently in Spain, 118 [7] centers provide radiation therapy services. Although its delivery is relatively standardized in our country, there's no data published about peer-review system in those departments so the extent and structure of this process vary widely among centers and even remain uncertain at some point.

In respect to our institution's peer-review process, we report the rate of plan changes as well as the amount of time and resources needed for this process. The aim of our study is to demonstrate the feasibility of performing peer review to every case in radiation therapy. A sample of typical results is presented just as illustrative purpose.

\section{Materials and methods}

Historically, peer review was established at our institution as an audit procedure to review treatment indication, target/OARs volumes, DVH analyses, treatment plan changes and any other remarkable aspects concerning patient's case, recorded in our quality guarantee department certification.

These conferences occur daily at 8.15 am where all patient cases are presented prior to dosimetry and treatment initiation.

The review process is performed by a multidisciplinary team that includes physicians, resident physicians and medical physicists. Although daily attendance varies, typically at least six attending physicians (range 2-10) are present at each session. The minimum number of senior radiation oncologists required to run the meeting is two, but this situation only occurs when vacation period, attendance to several tumor committees, previous day on-calls, or special considerations such as medical leave or congress attendance meet. After this conference, Radiophysics Department has its own specific meeting to discuss cases presented at our peer review among other topics. This conference is attended by medical physicists, physic residents and dosimetrists. We balanced this approach to respect the workflow of both departments.

During the conference, the treating physician or covering physician resident provides a brief review of the patient's history in addition to treatment intentions. Patient's history, physical exam findings, radiological images and documents such as anatomopathological analysis or complete surgical reports are available within electronic medical record and linked via computer to live broadcasting during discussion if questions arise.

Simultaneously, all axial, sagittal and coronal slices of patient's simulation CT scan and any image fusions (PET/ $\mathrm{CT}$ or MRI) are examined and projected streaming on a screen to review target volumes including gross tumor volume (GTV), clinical target volume (CTV) and planning target volume (PTV) as well as organ at risk (OAR) contours/ constraints using Aria ${ }^{\circledR}$ 15.5 Varian Medical System.

Peer-review activity regarding patient's identification, main case's characteristics and decisions made in our conference is recorded in an official logbook.

Individual DVH analysis and dosimetry in relation of PTV coverage and OAR sparing are reviewed only if case is considered complex (i.e., re-irradiation, cases involving re-planning or adaptive planning, pediatric, etc.). Cases 
presented must accomplish our department's precise established DVH/constraints protocol on each pathology to facilitate workflow and to highlight those cases requiring thorough peer review to achieve efficiency compromise and make peer review $100 \%$ feasible.

In cases of re-irradiation, composite plans with previous doses are examined during peer review.

Retrospectively, urgent treatments performed the previous day during on-calls are also presented. Controversial cases discussed at pretreatment review might be presented again before treatment initiation focusing on final approved plan, DVH and any remarkable issue on treatment delivery.

Attendees are encouraged to make suggestions during and after presentation of the case regarding aspects of the radiation treatment plan such as target volumes and normal tissue contours in close proximity to them, total dose, dose fractionation, frequency, treatment intention, technique, IGRT imaging or any other details with implication in treatment planning or dose calculation (i.e., skin affectation or not, prosthesis, setup margins, DIBH, pacemaker and its relevance, photon/electron boost radiotherapy, registration quality, PTVs coverage and OAR constrains...).

Cases requiring special procedures (brachytherapy, radiosurgery, total body irradiation, intra-operative radiation therapy) are currently being revised by groups with experience in those techniques prior to treatment delivery and only controversial treatment indications (i.e., salvage treatment with brachytherapy for irradiated patients) or cases in which boost with HDR-BT is going to be performed after EBRT (i.e., breast cancer, cervical cancer) go through our peer-review conferences before starting treatment.

In addition, as a Department with in-trainee physicians, peer-review conference serves as a forum for continuing education to collectively analyze every case to recognize proper diagnosis procedures, previous/concurrent chemotherapy, data from previous surgery, possible future oncologic procedures and indication or not for radiation therapy treatment.

Collectively, plans are either accepted or rejected with indication of new presentation. Cases are classified into four categories: no changes, minor changes, major changes or plan rejection. Parameters analyzed to determine change's nature are the same for minor/major: contouring, dose/ fractionation, technique or a combination. Criteria to allocate a recommended change into minor or major takes into account the clinical impact of the plan on each patient. If approvement is achieved, then the plan does not have to go back through conference (i.e., no changes, minor/major change recommended), but if it was rejected for re-planning, then the case must be presented again according the agreed change level. Changes can be done by the planner in real time (i.e., minor/major changes to total dose or dose fractionation) or can be performed later (i.e., minor/major changes in contouring).

When a plan is rejected, usually a combination of circumstances might be present (i.e., more than one major change, a decision to present the case in tumor committee for neo/ adjuvant chemotherapy or immunotherapy, an indication to perform a new simulation CT scan for newly discoveries during peer review or for a better patient's immobilization, no treatment decision, a suggested confirmation of dose/ fractionation/constraints in a certain location for SBRT...).

Modifications made after our conference to radiation therapy plans are documented by the attendance medical physicist. Dosimetry can only be assessed if changes suggested in peer review are made by the responsible radiation oncologist and therefore compliance with the conference recommendation before treatment initiation is ensured.

Based on our group peer-review process, we prospectively recollected some data on cases presented at our institutional conference to quantify the rate of plan changes, time and resources needed for this process. Items such as time to present per case, type of patient (adult or pediatric), treatment intent, tumor type (primary, metastasis or both), dose, technique, disease location and receipt of previous radiation were gathered. Cases were then analyzed to determine the rate of major change, minor change and plan rejection after presentation as well as the type of change (dose/fractionation, contouring, technique or combination) and also median time per session.

\section{Results}

Over a period of 4 weeks 148 cases underwent peer review at our department prior to starting treatment. A total of 20 conferences took place during this period. Median of attendants was six physicians (range 2-10), three in-training-physicians (range 1-4) and one medical physicist.

Approach assessment was the most frequent reason for case presentation (94.6\%).

The majority of cases presented were adult patients (97.3\%). In terms of type of tumor, $66.2 \%$ were primary tumors and $32.4 \%$ were metastasis. The most common technique proposed in the plans review was 3D-CRT which was performed in approximately $44 \%$ of cases presented, followed by VMAT (37.2\%), SBRT (17.6\%) and SR (1.3\%). $47.3 \%$ of cases had a radical treatment intent, $38.5 \%$ had a neo/adjuvant treatment intent and $12.8 \%$ of cases were presented for palliation treatment. Nearly $22 \%$ of presented cases had received previous radiation nearby the area of concern, in-field or at other location either at our institution or another institution. The most frequently reviewed sites were breast (30.4\%), followed by pelvis (18.2\%), bone/soft tissue 
(14.9\%) and central nervous system (14.2\%). A summary of characteristics of cases presented is found in Table 1.

As previously described, once the plan is reviewed at the conference it can be approved or rejected for re-planning if it undergoes a plan rejection. In our case, nearly $80 \%$ of cases were accepted without changes, $11.5 \%$ with minor changes, $6 \%$ with major changes and 3.4\% were rejected with indication of new presentation. Most frequent reason of change was contouring corrections $(53.8 \%)$ followed by dose or fractionation (26.9\%). Peer review resulted in breast (29\%), pelvis (22.6\%), bone/soft tissue (16.1\%) and H\&N (16.1\%) cases requiring most of the changes recommended. $96.9 \%$ of cases were accepted after presentation. Overall, total rate of change including both major and minor changes and plan rejection was nearly $21 \%$. A summary of the change rates is found in Table 2.

Furthermore, median time per session was 38 min (range 4-72). The majority of cases (59.5\%) were presented in $1-4 \mathrm{~min}, 32.4 \%$ in $5-9 \mathrm{~min}$ and $8.1 \%$ in $\geq 10 \mathrm{~min}$. Of the sites presented, the longest median time of presentation was for abdomen cases $(8 \mathrm{~min})$, followed by pelvis $(6 \mathrm{~min})$ and central nervous system (6 min). In terms of technique, SR (10 $\mathrm{min})$ and SBRT (6 min) had the longest mean time of presentation. Additionally, re-irradiation cases, no treatment decision cases discussed and those that had any type of change had slightly longer presentation times. A summary of presentation's length is found in both Tables 1 and 2.

\section{Discussion}

This study analyzes peer-review process at our institution and its feasible implementation to our daily clinical practice. A remarkable proportion of modifications have potential clinical implications as disease outcome could be directly affected by radiation therapy plan [8]. Introducing this 'audit and feedback' process among each center's protocol is a quality assessment whereby adherence to published guidelines is measured $[9,10]$. We found a total change rate of $20.9 \%$ of all cases presented and a major change rate of $6 \%$. Previous studies identified similar proportions of recommended plan changes after peer review, accounting for 23.3 [6], 26 [11], 22.4 [12], 12.2 [13] and $10.8 \%$ [14] of total change rate and a major plan change rate of 8.2 [6], 8.6 [12], 2.6 [13], 1.8 [14] and 27\% [11] respectively. In addition, some reviews also included changes in total dose $(16.4 \%)$ or fractionation $(6.8 \%)$ [6] that did not resulted in re-planning and a total minor change rate of 37 [11], 12.9 [12],7.3 [14] and $47.8 \%$ [15] respectively, which is in alignment with the $11.5 \%$ rate of minor changes obtained at our institution. Accordingly to a survey of North American teaching
Table 1 Summary of characteristics of cases presented

\begin{tabular}{|c|c|c|}
\hline Characteristic & $\begin{array}{l}\text { Number of cases } \\
(n=148)\end{array}$ & $\begin{array}{l}\text { Mean time of } \\
\text { presentation } \\
(\mathrm{min})\end{array}$ \\
\hline \multicolumn{3}{|l|}{ Site } \\
\hline Bone/soft tissue & $22(14.9 \%)$ & 5 \\
\hline CNS & $21(14.2 \%)$ & 6 \\
\hline Breast & $45(30.4 \%)$ & 3 \\
\hline Abdomen & $6(4.1 \%)$ & 8 \\
\hline Pelvis & $27(18.2 \%)$ & 6 \\
\hline GU & $14(9.4 \%)$ & 6 \\
\hline GI & $5(3.4 \%)$ & 5 \\
\hline GYN & $6(4.1 \%)$ & 5 \\
\hline Other & $2(1.3 \%)$ & 7 \\
\hline Head and neck & $7(4.7 \%)$ & 4 \\
\hline Thorax & $20(13.5 \%)$ & 5 \\
\hline Lung & $18(12.2 \%)$ & \\
\hline Other & $2(1.3 \%)$ & 5 \\
\hline \multicolumn{3}{|l|}{ Age } \\
\hline Adult & $144(97.3 \%)$ & 5 \\
\hline Pediatric & $4(2.7 \%)$ & 6 \\
\hline \multicolumn{3}{|l|}{ Motive } \\
\hline Indication & $6(4.1 \%)$ & 7 \\
\hline Approach & $140(94.6 \%)$ & 5 \\
\hline Not-expected evolution & $2(1.3 \%)$ & 6 \\
\hline \multicolumn{3}{|l|}{ Type of tumor } \\
\hline Primary & $98(66.2 \%)$ & 4 \\
\hline Metastasis & $48(32.4 \%)$ & 6 \\
\hline Both & $2(1.3 \%)$ & 15 \\
\hline \multicolumn{3}{|l|}{ Treatment intent } \\
\hline Radical & $70(47.3 \%)$ & 5 \\
\hline Palliative & $19(12.8 \%)$ & 4 \\
\hline Neo/Adjuvant & $57(38.5 \%)$ & 4 \\
\hline No treatment & $2(1.3 \%)$ & 6 \\
\hline \multicolumn{3}{|l|}{ Technique } \\
\hline 3D-CRT & $65(43.9 \%)$ & 3 \\
\hline VMAT & $55(37.2 \%)$ & 5 \\
\hline SBRT & $26(17.6 \%)$ & 6 \\
\hline SR & $2(1.3 \%)$ & 10 \\
\hline \multicolumn{3}{|c|}{ Receipt of previous radiation } \\
\hline No & $116(78.4 \%)$ & 4 \\
\hline In-field & $6(4.1 \%)$ & 4 \\
\hline Near & $6(4.1 \%)$ & 9 \\
\hline Other location & $20(13.4 \%)$ & 5 \\
\hline \multicolumn{3}{|l|}{ Length of presentation } \\
\hline $1-4 \min$ & $88(59.5 \%)$ & - \\
\hline $5-9 \min$ & $48(32.4 \%)$ & - \\
\hline$\geq 10 \min$ & $12(8.1 \%)$ & - \\
\hline
\end{tabular}

$C N S$ central nervous system, $G U$ genitourinary, GI gastrointestinal, $G Y N$ gynecologic, $3 D$-CRT three-dimensional conformal radiation therapy, VMAT volumetric modulated arc therapy, SBRT stereotactic body radiation therapy, $S R$ stereotactic radiosurgery 
Table 2 Rate of plan changes/rejection after presentation

\begin{tabular}{|c|c|c|}
\hline Type of change & $\begin{array}{l}\text { Number of cases } \\
(n=148)\end{array}$ & $\begin{array}{l}\text { Mean time of } \\
\text { presentation } \\
(\mathrm{min})\end{array}$ \\
\hline \multicolumn{3}{|l|}{ Any change } \\
\hline Yes & $31(20.9 \%)$ & 7 \\
\hline No & $117(79.1 \%)$ & 4 \\
\hline \multicolumn{3}{|l|}{ Acceptation } \\
\hline Yes & $143(96.6 \%)$ & 4 \\
\hline No & $5(3.4 \%)$ & 12 \\
\hline \multicolumn{3}{|l|}{ Changes' categories } \\
\hline Minor & $17(11.5 \%)$ & 5 \\
\hline Major & $9(6 \%)$ & 6 \\
\hline \multicolumn{3}{|l|}{ Type of change } \\
\hline Dose/fractionation & $7(26.9 \%)$ & 6 \\
\hline Contouring & $14(53.8 \%)$ & 5 \\
\hline Technique & $2(7.7 \%)$ & 4 \\
\hline Combination & $3(11.5 \%)$ & 5 \\
\hline \multicolumn{3}{|l|}{ Changes' by site } \\
\hline Bone/soft tissue & $5(16.1 \%)$ & 7 \\
\hline CNS & $2(6.5 \%)$ & 8 \\
\hline Breast & $9(29 \%)$ & 5 \\
\hline Pelvis & $7(22.6 \%)$ & 7 \\
\hline GU & $2(6.5 \%)$ & 10 \\
\hline GYN & $3(9.6 \%)$ & 6 \\
\hline Other & $2(6.5 \%)$ & 7 \\
\hline Abdomen & $2(6.5 \%)$ & 9 \\
\hline Head and Neck & $5(16.1 \%)$ & 6 \\
\hline Thorax & $1(3.2 \%)$ & 7 \\
\hline Lung & $1(3.2 \%)$ & 7 \\
\hline
\end{tabular}

$C N S$ central nervous system, $G U$ genitourinary, $G Y N$ gynecologic

centers, $75 \%$ of respondents estimated that major changes occurred in $<10 \%$ whilst minor changes were estimated to be requested in $<10 \%$ of cases by $61 \%$ of respondents [5]. Contouring corrections (53.8\%) followed by dose/fractionation $(26.9 \%)$ resulted in most frequent reason of change, similarly to data reported in other reviews $[6,12-14,16]$. It is true that contouring plays an important role in our revision because it is known to be a major responsible for treatment outcomes and also isodose or DVH data are completely dependent on contour definition, especially when using IMRT-VMAT.

To optimize peer-review process, Ballo et al. [13] highlighted the importance of reporting disease sites to identify those with a higher proportion of changes resulting in $\mathrm{H} \& \mathrm{~N}$, GI and GYN cases requiring a plan change more than $15 \%$ of the time. Esophagus, uterus, upper/lower limb, cervix, $\mathrm{H} \& \mathrm{~N}$, bilateral lung, right supraclavicular lymph nodes, rectum and spine were cancer groups proposed to most necessitating mandatory peer review according to Rouette et al.
[15]. We found breast (29\%), pelvis (22.6\%), bone/soft tissue $(16.1 \%)$ and $\mathrm{H} \& \mathrm{~N}(16.1 \%)$ cases requiring most of the changes recommended from our peer-review members during our monthly recruitment period.

Several different models have been suggested for peerreview process within radiation oncology. A survey developed by ASTRO to its physician members revealed considerable variations among centers in the timing and content of peer review requiring additional clear guidance and formal recommendations from expert consensus [2]. Some practices have established a multistep process with separate peerreview stages consisting of a first presenting case reunion followed by consensus planning conferences and final chart rounds to evaluate patients who have started a new treatment plan. Other institutions have a dual-layer process with both early and late peer review evaluating different aspects. In addition, centers perform either prospective peer review (before radiation) or once the therapy has started (inside the first week) and mostly discuss only cases considered complex [2, 3, 6, 11]. According to RD 1566/1988 [17], items such as treatment indication, contouring, isodoses, DVH and not expected reactions should be discussed in departmental clinical sessions. At our institution, peer-review process is unique: in a single conference we always gather the first two aspects of cases mentioned above and the third one when case is considered complex due to a strict established service protocol, and then modify plans when indicated based on peers recommendations always prior to treatment initiation, which is considered beneficial and easier to implement. The fourth aspect is not routinely assessed and we might contemplate including it henceforth as an additional quality control of the radiation therapy process complying with established protocols endorsed by scientific societies of recognized solvency such as ASTRO and ESTRO.

Notably, most centers focus on peer reviewing EBRT cases leaving special treatment modalities (brachytherapy, stereotactic radiosurgery) revision rates to a significantly lower proportion. In particular, Lawrence et al. [5] detected that $39 \%$ of institutions across the United States never peer review pre-implant or post-implant dosimetry for prostate brachytherapy, 29\% never peer review gynecologic brachytherapy cases and only $58 \%$ consider RS. Also, Hoopes et al. [2] pointed out that prospective review in brachytherapy cases accounted for $30 \%$ among physicians surveyed.

It is remarkable to mention that, as a reference center for brachytherapy procedures at Valencian Community and at national level, we treat patients from different institutions who have already received EBRT or will be sent back to complete it after our brachytherapy treatment it's performed. Although each brachytherapy procedure is unique for us, currently final treated plans are unfortunately not routinely presented at our daily peer-review conference. 
In agreement with Lawrence et al. [5], brachytherapy is of potential concern because it involves high-dose radiation with curative intent in which spatial issues (applicator position, target definition) can have highly significant consequences. In our opinion, each brachytherapy procedure is unique, in the sense that depends not only on the patient conditions but also on the physician skills training, hence all brachytherapy cases might retrospectively be reviewed To evaluate the quality of the implants, fiducial marker placement, the problems and solutions found, etc. This should complete our quality control system protocol and a forthcoming event at our department along with other special treatment modalities (stereotactic radiosurgery, total body irradiation, intra-operative radiation therapy...) on which controversial treatment indications are presented but technical considerations and final treated plan should be retrospectively review for educational benefits and treatment planning consistency, reinforcing collective decision making and assuring quality control, with further investigation into its clinical impact.

Interestingly, the variability of parameters evaluated in the classification for minor/major changes and therefore its implication in the acceptation/rejection plan rates it's still an unsolved question as there's no specific guidance published from professional organizations [1]. This inconsistency can be appreciated through previous articles. For example, to Ballo et al. [13], events variations are classified in three types: radiation dose, target or major changes that changed treatment modality (i.e., surgery rather than external beam radiation) or added modalities (i.e., chemoradiation therapy rather than radiation alone). Driving forward into changes' nature, Rouette et al. [15] analysis defines a minor change as a recommended change that did not meet the criteria for a "major" change and did not lead to significant repeat treatment planning whilst a major change is defined as a change requiring repeat planning and/or have a foreseeable effect on treatment toxicity or cancer outcomes in the view of the peer-review physician, which is more in alignment with our change's proposal.

During our monthly prospective data base, we conducted major changes in nine cases $(6 \%)$ and minor changes in 17 cases (11.5\%). Major recommendations at our multidisciplinary conference accounted for contouring (i.e., breast cancer case involving regional lymph nodes that have not been considered when contouring before peer review or modifications to GTV), dose/fractionation (i.e., changes in dose/fractionation for SBRT-VMAT lung case from $12 \times 5$ Gy to $1 \times 36$ Gy or unacceptable and avoidable OAR dose in a re-irradiation pelvic case) and technique (i.e., 3D-CRT instead of VMAT for soft tissue sarcoma of the limb) while a change in dose/fractionation for palliative bone metastasis from $10 \times 3$ Gy to $5 \times 4$ Gy due to patient's performance status was considered a minor change and adding PRV to a certain OAR was considered a minor contouring recommendation.

From our point of view and awaiting for more official detailed guidance, the definition of recommendation magnitude to classify a change into minor or major is inherently subjective and should be appraise individually on each case and assigned to every radiation oncology department itself.

Arising from our experience, peer-review process brings to light unperceived considerations or additional viewpoints that could lead to changes resulting in the outcome of radiation plans that otherwise would not have been made without this process. The degree of compliance with peerreview recommendations arising from Walburn et al. [11] was reasonably good (59\%) but it decreased as recommendation magnitude increased: $65 \%$ for minor and $47 \%$ for major recommendations respectively, suggesting that early rather than late peer review may enhance compliance and efficiency creating a safety-oriented culture. Moreover, Rouette et al. [15] reported a rate of $38.5 \%$ of major changes for plans presented after treatment had begun. Fairchild et al. [8] revealed that retrospective peer review resulted in higher failure rates and worse overall and progression-free survival after delivery of radiation that did not make compliant with protocol guidelines. Consequently, along with ASTRO [1] we recommend performing peer review before the initiation of treatment for assuring plan quality and patient safety.

Additionally, an important overarching question remains concerning the optimal proportion of plans to review to optimize the utility of peer review. In our conferences, we present every single patient and not only those cases considered complex, explaining why breast cancer accounted for $30.4 \%$ and therefore why 3D-CRT was the most common technique utilized (43.9\%) due to the high volume of patients suffering from this pathology and to palliative cases.

Previous publications suggested that peer review might not be necessary on every breast cancer patient [13].From our point of view, breast cancer is of great importance as radiotherapy is considered a radical treatment in this pathology with significant curative rates and arising controversies among axillary management. Although wide guidance recommendations published by experts help reducing practice variation, we have a high level of rigorousness when performing CT-scan-based contouring and, for patients with nodal involvement, the CT slice that will establish the separation between the beams treating breast (tangential beams) and the half beams treating nodes is also decided in our peer-review conference among radiotherapists and with the advice of the medical physicist. This decision has a huge impact in the reduction of lung dose, humeral head avoidance, spinal cord and medial extension of nodal PTV as well as an important reduction of the planning time.

Following on from this, Lymberiou et al. [18] showed that from 2223 breast radiation plans analyzed $4.4 \%$ required 
major changes while Rouette et al. [15] identified a rate change of $41.5 \%$ for left/right breast plans and $30.8 \%$ for left/right chest wall. Both studies noticed that plans involving the regional lymph nodes were more likely to have recommended changes suggesting that cases with locoregional nodal involvement should be a significant area of focus for peer review.

Moreover, from 148 cases presented at our conference during that monthly period, seven accounted for $\mathrm{H} \& \mathrm{~N}$ cases $(4.7 \%)$, with a reported change rate of $16.1 \%$ from total changes. The majority of these changes were considered minor (dose/fractionation or contouring) and only $6.5 \%$ were considered major enough to affect clinical care (i.e., one case was rejected with indication of presentation in the H\&N tumor committee to considered neoadjuvant chemotherapy).

As described in previous studies, $\mathrm{H} \& \mathrm{~N}$ is a frequent revision's site mainly because it's inherent complexity in normal tissue contouring but also because there are always physician-specific decisions that are only exposed during a process of peer review [13].

Rouette et al. [15] compiled 5561 peer-reviewed treatment plans among 14 cancer centers in Ontario including $714 \mathrm{H} \& \mathrm{~N}$ cases $(12.8 \%)$ with changes recommended after peer review in $5.9 \%$. Of the 2830 cases presented over a period of 4 years of peer review at Ballo et all analysis [13], 421 were $H \& N$ patients with a reported change rate of $34.2 \%$, prompting that peer review should be mandatory for all $\mathrm{H} \& \mathrm{~N}$ plans and prior to treatment initiation in favor of re-planning avoidance.

Similarly to our proportion of cases peer reviewed, Albert et al. [6] found $15 \mathrm{H} \& \mathrm{~N}$ cases (20.5\%) from an overall of 73 cases presented resulting in one of the most frequently reviewed sites and also with one of the longest mean time of presentation (9 $\mathrm{min})$.

Discussing all patients might seem to lengthen the time needed for peer-review process and also could led to an unnecessary effort, but when comparing our median time per session (38 $\mathrm{min}$ ) to other experiences [5, 6], the result is quite similar. Based on site involvement, previous experiences reported differences in the time needed to present cases. Albert et al. [6] found that cases involving lung, CNS and $\mathrm{H} \& \mathrm{~N}$ required slightly more time whereas Ballo et al. [13] suggested performing peer review for all disease sites but focusing on those requiring changes more often $(\mathrm{H} \& \mathrm{~N}$, GI or GYN). For us, abdomen cases, pelvis (especially GU, GYN) and CNS accounted for the longest mean time of presentation likely due to SBRT cases with critical structures nearby and higher number of OARs reviewed for these sites.

Ultimately, radiation oncology departments should integrate peer-review system into their routine workflow, scheduling conferences at the optimal moment of the day and with a reasonable average of reunions per week to reassure staff that their time is well spent.
Furthermore, peer review serves for educational opportunities and creates an environment that fosters respectful questioning. Resident physicians have the opportunity to take an active part in the process by asking questions and giving their own feedback, becoming that way proficient in plan evaluation. Seasoned physicians can also benefit from peer review given the variety of cases presented, maintaining them updated for disease sites plans even if they are not their main sites of focus [6]. Topics arising from clinical cases on peer review are also adopted to create academic bibliographical sessions every 2 weeks approximately reinforcing its importance to stay abreast of the latest updates in the oncology field. In addition, this system has also indirect quality benefits: reduces practice variation by promoting standardization, improves communication and reinforces knowledge sharing [19]. It has also been documented that centralized plan review increases group consensus and consistency which is reflected in decreased treatment plan changes over time, suggesting that the act of peer review has an educational effect and the system directly change care by increasing adherence to institutional guidelines [13]. All of this enhance the role and utility of peer review as part of routine clinical practices.

Nevertheless, despite evidences providing proof of its benefits, previous surveys showed that only approximately $50 \%$ of centers performed peer review of radiation plans in the United States [2] and only 52.3\% in Canada [19].

In Europe, harmonizing clinical care based on expert consensus agreement can improve clinical outcome in the face of uncertainty, with quality and safety playing a priority place in radiation medicine according to ESTRO [20]. Recently, the Working Group on Patient Safety and Quality of the Spanish Society of Radiation Oncology (SEOR) revised national and international recommendations for patient safety evaluating whether they are included in Spanish legislation. They found that even though peer review plays an important part in maintaining quality standards and improving performance and safety of care, neither RD 1566/1988 nor RD 815/2001 consider the peer-review process [21]. Nevertheless, a new RD 601/2019 [22] reinforcing mandatory OARs and treatment volumes revision became effective a month ago so performing peer review will be a compelling reason henceforth. Unfortunately, there's no data published about peer-review system in Spain so far to our knowledge. This report is the first one providing information about the structure, operation and metrics analyzed in this process at national level.

Finally, advances in tumor genomic data and technology will enable more personalized radiation treatment based on patient-specific knowledge. In this context, artificial intelligence (AI) will be a part of our future by providing prognostic models built on radiomic features, creating automated segmentation of OARs/TVs on contouring 
area so as to prediction of normal tissue complications and assuring quality by streamline physician peer-review process. Even though potential benefits of AI in radiation quality assurance, challenges remain before its widespread implementation in clinical setting [23, 24]. From our point of view, adopting contouring guidance similar to those used in clinical trials seems to be essential nowadays in the delivery of radiation treatment to assure quality as an established methodology exists.

We believe peer review is a quality assurance procedure to guarantee patient safety and feasible within the structure of every Radiation Oncology Department.

Emergently, due to COVID-19 outbreak, drastic disease-control measures from official institutions have forced centers to adapt their usual clinical care and organization level [25]. On this scenario, teleconferencing via WebEx ${ }^{\circledR}$ has been established to allow staff to conduct peer review and, therefore, maintaining QA and patient's security in spite of the situation.

There are several weaknesses to consider within our current analysis, but by publishing this review we would like to encourage other centers to implement peer review to their daily routine and to report their experiences proving its feasibility.

One limitation is the low sample of plans compiled (148) compared to other publications with thousands [11, $13,15,26]$. As we revise all cases, data collected represents an assortment of pathologies treated at our institution during a whole month period and, even though we assume it is not accurate to extrapolate results, we believe it gives the reader an idea of the amount of cases and disease's locations presented during a year (1701 EBRT cases in 2019).

Secondly, not expected reactions and retrospective peer review to other special modalities (brachytherapy, SR, TBI, IORT...) are not routinely assessed and seems to be a compelling inclusion henceforth for assuring additional QA system and its impact in clinical outcomes.

\section{Conclusion}

Our findings show that everyday group consensus peer review is an efficient manner to recollect clinical and technical data of cases presented to ensure quality radiation care before initiation of treatment as well as creating a feedback team environment. It is also considered as a security system to avoid potential errors whereas serving for continuing education. Despite the challenge nature of its implementation, our model demonstrates its feasibility within the normal operating of every Radiation Oncology Department.
Funding Not applicable.

\section{Compliance with ethical standards}

Conflict of interest Authors declare that there is no conflict of interests regarding the publication of this paper.

Research involving human participants and/or animals Not applicable.

Informed consent Not applicable.

\section{References}

1. Marks LB, Adams RC, Pawlicki T, et al. Enhancing the role of case-oriented peer review to improve quality and safety in radiation oncology: executive summary. Pract Radiat Oncol. 2013;3:149-56.

2. Hoopes DJ, Johnstone PA, Chapin PS, et al. Practice patterns for peer review in radiation oncology. Pract Radiat Oncol. 2015;5(1):32-8

3. Duggar WN, Bhandari R, Yang CC, et al. Group consensus peer review in radiation oncology: commitment to quality. Radiat Oncol. 2018;13:55.

4. Brundage M, Foxcroft S, McGowan T, et al. A survey of radiation treatment planning peer review activities in a provincial radiation oncology programme: current practice and future directions. BMJ Open. 2013;3:e003241.

5. Lawrence YR, Whiton MA, Symon Z, et al. Quality assurance peer review chart rounds in 2011: a survey of academic institutions in the United States. Int J Radiat Onco Biol Phys. 2012;84(3):590-5.

6. Albert AA, Duggar WN, Bhandari R, et al. Analysis of a real time group consensus peer review process in radiation oncology: an evaluation of effectiveness and feasibility. Radiat Oncol. 2018;13:239.

7. Rodriguez A, Algara M, Monge D, et al. Infrastructure and equipment for radiation oncology in the Spanish National Health System: analysis of external beam radiotherapy 2015-2020. Clin Transl Oncol. 2018;20(3):402-10.

8. Fairchild A, Straube W, Laurie F, et al. Does quality of radiation therapy predict outcomes of multi center cooperative group trials? A literature review. Int J Radiat Onco Biol Phys. 2013;87:246-60.

9. Hanks GE. Quality control and assurance. Am J Clin Oncol. 1998;11:411-4.

10. Levitt SH, Khan F. Quality assurance in radiation oncology. Cancer. 1994;74(Suppl 9):2642-6.

11. Walburn T, Wang K, Sud S, et al. A prospective analysis of radiation oncologist compliance with early peer review recommendations. Int J Radiat Onco Biol Phys. 2019;104(3):494-500.

12. Qureshi BM, Mansha MA, Karim MU, et al. Impact of peer review in the radiation treatment planning process: experience of a tertiary care university hospital in Pakistan. J Glob Oncol. 2019;5:1-7.

13. Ballo MT, Chronowsky GM, Schlembach PJ, et al. Prospective peer review quality assurance for outpatient radiation therapy. Pract Radiat Oncol. 2014;4(5):279-84.

14. Brunskill K, Nguyen TK, Boldt RG, et al. Does peer review of radiation plans affect clinical care? A systematic review of the literature. Int J Radiat Oncol Phys. 2017;97(1):27-34.

15. Rouette J, Gutierrez E, O'Donnell J, et al. Directly improving the quality of radiation treatment through peer review: a 
cross-sectional analysis of cancer centers across a provincial cancer program. Int J Radiat Onco Biol Phys. 2017;98(3):521-9.

16. Boxer M, Forstner D, Kneebone A, et al. Impact of a real-time peer review audit on patient management in a radiation oncology department. J Med Imaging Radiat Oncol. 2009;54(4):405-11.

17. Real Decreto $1566 / 1998$, de 17 de julio, por el que se establecen los criterios de calidad en radioterapia. BOE núm. 206, de 28 de agosto de 1998, páginas 29383 a 29394.

18. Lymberiou T, Galuska S, Lee G. Predictors of breast radiotherapy plan modifications: quality assurance rounds in a large cancer centre. Radiother Oncol. 2015;114:17-211.

19. Caissie A, Rouette J, Jugpal P, et al. A Pan-Canadian survey of peer review practices in radiation oncology. Pract Radiat Oncol. 2016;6:342-51.

20. Coffey M, Leech M. The European Society of Radiotherapy and Oncology (ESTRO) European higher education area levels 7 and 8 postgraduate benchmarking document for Radiation TherapisTs (RTTs). Tech Innov Patient Support Radiat Oncol. 2018;8:22-40.

21. Pardo MJ, del Cerro PE, Contreras MJ, et al. Patient safety in radiation oncology in Spain: a need to change. Clin Transl Oncol. 2020;22:751-8.

22. Real Decreto 815/2001, de 13 de julio, sobre justificación del uso de las radiaciones ionizantes para la protección radiológica de las personas con ocasión de exposiciones médicas. BOE 168, de 14 de Julio de 2001, páginas 25591 a 25594.

23. Kiser KJ, Fuller CD, Reed VK. Artificial intelligence in radiation oncology treatment planning: a brief overview. J Med Artif Intell. 2019;2:9.

24. Thompson RF, Valdes G, Fuller CD, et al. The future of artificial intelligence in Radiation Oncology. Int J Radiat Oncol Bill Phys. 2018;102(2):247-8.

25. Simcock R, Thomas TV, Estes C, et al. COVID-19: global radiation oncology's targeted response for pandemic preparedness. Clin Transl Radiat Oncol. 2020;22:55-68.

26. Surucu M, Bajaj A, Roeske JC, et al. The impact of transitioning to prospective contouring and planning rounds as peer review. Adv Radiat Oncol. 2019;4(3):532-40.

Publisher's Note Springer Nature remains neutral with regard to jurisdictional claims in published maps and institutional affiliations. 